\title{
Clinical Study \\ Benefit of Leukocyte- and Platelet-Rich Plasma in Operative Wound Closure in Oral and Maxillofacial Surgery
}

\author{
Agata Cieślik-Bielecka, ${ }^{1}$ Justyna Glik, ${ }^{2}$ Rafał Skowroński, ${ }^{3}$ and Tomasz Bielecki ${ }^{4}$ \\ ${ }^{1}$ Department of Maxillofacial Surgery, Medical University of Silesia, Sosnowiec, Poland \\ ${ }^{2}$ Burn Wound Unit, Burn Center, Siemianowice Slaskie, Poland \\ ${ }^{3}$ Orthopedic Surgery and Traumatology Department, Medical University of Białystok, Białystok, Poland \\ ${ }^{4}$ Department and Clinic of Orthopaedics, Trauma and Reconstructive Surgery, Trauma Center St. Barbara Hospital, \\ Medical University of Silesia, Sosnowiec, Poland \\ Correspondence should be addressed to Tomasz Bielecki; tomekbiel@o2.pl
}

Received 9 June 2016; Accepted 13 November 2016

Academic Editor: Adriano Piattelli

Copyright (C) 2016 Agata Cieślik-Bielecka et al. This is an open access article distributed under the Creative Commons Attribution License, which permits unrestricted use, distribution, and reproduction in any medium, provided the original work is properly cited.

\begin{abstract}
This article reports the influence of an autologous leukocyte- and platelet-rich plasma (L-PRP) injection as a minimally invasive method on supporting wound healing processes after a mandibular odontogenic cystectomy and double mandibular fracture fixation. 113 patients were enrolled into a control group (received no L-PRP injection) and 102 patients were enrolled into an L-PRP group with an oral mucosa incision. 18 patients after a double mandibular fracture were operated on using 2 external submandibular approaches receiving no fluids in the right site (a control group) and an L-PRP injection in the left incision (L-PRP group). Clinical observations showed that the oral mucosa healed faster in patients treated with L-PRP, in comparison to cases where inductive biomaterial was not added. Pain at the L-PRP injection site was relieved within few hours after an operation in patients with double mandibular fractures. However, there were no differences observed in the progression of the healing process. L-PRP possesses inductive properties that could stimulate healing processes and it seems to be one of the most promising methods in the future for the treatment of soft tissue defects.
\end{abstract}

\section{Introduction}

Recent advances in cellular and molecular biology have led to the identification of specific cytokines that mediate cellular activities [1-3]. The ability to control cellular activities becomes a powerful tool in the management of surgical disorders. Platelets retain numerous growth factors such as platelet derived growth factor (PDGF), transforming growth factor beta (TGF- $\beta$ ), insulin-like growth factors I and II (IGF I and IGF II), and epidermal growth factor (EGF) $[4,5]$. By concentrating platelets, higher levels of growth factors can be obtained. Amongst others, autologous leukocyte- and platelet-rich plasma (L-PRP) enriched with growth factors may enhance new tissue formation in vitro $[1,2,6]$ and in vivo $[3,7]$.

For years, it was considered that the properties of the concentrates were mainly related to the high concentrations of platelet derived growth factors in their composition.
However, several authors started to insist on the key role of fibrin architecture and leukocytes within adult stem cell content of these preparations, and they also started to develop the concept that the growth factors alone cannot be considered as the main players of these products $[3,7]$,

In recent years, the application of leukocyte- and platelet-rich plasma (L-PRP) to enhance bone regeneration and soft tissue maturation has increased chronic healing disturbances and many attempts to explain L-PRP properties of stimulating and accelerating tissue healing have been made [2]. Sprayed or percutaneous administration of L-PRP with inductive properties into wound closure disturbances offers the advantage of decreasing morbidity that is associated with the widely used classic open grafting technique. However, L-PRP injections into the wound during the first phase of the healing process in the maxillofacial operation procedure were written incidentally. 
This article reports the influence of a percutaneous autologous L-PRP injection as a minimally invasive method for the additional treatment of surgical wounds after a mandibular cystectomy and double mandibular fracture fixation.

\section{Material and Methods}

A total of 233 patients from the Department and Clinic of Oral and Maxillofacial Surgery and outpatient clinic in Zabrze (Poland) were enrolled in the study; the following criteria were used to recruit participants to this study:

(1) diagnosis on the basis of radiological examinations an odontogenic mandibular cyst (mucosa group);

(2) diagnosis on the basis of radiological examinations a double mandibular fracture that qualifies for fixation by external approaches (skin group);

(3) no previous surgical treatment of the submandibular region operation or cyst site;

(4) a state of good health;

(5) no evidence of infection or inflammation;

(6) regular attendance at the control visits.

These patients were divided into two groups; group 1 included patients with the presence of mandibular odontogenic cystectomy and group 2 included patients with double mandibular fracture. In the first group, the control group consisted of 113 patients treated operatively without LPRP application (group la). In the experimental group (102 patients), L-PRP was injected into the bone defect and under the mucosa after cyst removal (group 1b).

In group 2, 18 patients after a double mandibular fracture fixation by 2 external approaches received no fluids in the right surgical site (group 2a) but an L-PRP injection in the left incision (group 2b).

For estimating wound healing processes, each participant was followed up on a regular outpatient basis with clinical and Visual Analog Scale (VAS) examinations. All examinations were performed at day 0 and 1, 2, 3, 4, 5, 6, 7, 9, and 14 days after an operation.

The study was approved by the Ethics Committee of the Medical University of Silesia (Katowice, Poland) and informed consent was obtained from patients.

Gelling L-PRP Preparation and Surgical Procedure. This study used the GPS II system (Platelet Concentration System, Biomet, Warsaw, IN). Six milliliters of anticoagulant citrate dextrose solution was drawn into a $60 \mathrm{~mL}$ syringe, and after transferring it, $54 \mathrm{~mL}$ of venous blood was then drawn into the syringe. The total syringe volume was transferred into a sterile tube. The tube was centrifuged for 15 minutes at $3200 \mathrm{rpm}$. During this process blood was separated into 3 basic fractions: red blood cells, leukocyte- and plateletrich plasma (a "buffy coat"), and acellular plasma (AP). Six milliliters of the autologous platelet concentrate was obtained and drawn with a $10 \mathrm{~mL}$ syringe and $1,5 \mathrm{~mL} 1600 \mathrm{U} / \mathrm{mL}$ bovine thrombin in a $10 \%$ calcium chloride solution was drawn with

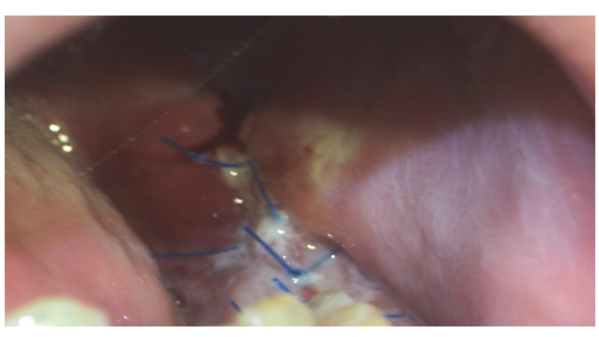

FIGURE 1: Postoperative wound in the 3rd day after cyst operationcontrol group.

a 2-mL syringe. Both syringes were then attached to the applicator tip.

The surgical procedure was carried out in the operating room under general anesthesia. A cystectomy was performed in the mucosa group and an internal stabilization in the skin group. Well-experienced surgical team operated on all patients. A double needle was used to form gelatinous mass in surgical site: bone cavity in mucosa group or operative wound in skin group. In the control groups, a gelling L-PRP was not given.

2.1. Statistical Analysis. Statistical analysis was performed using Statistica for Windows 6.1 version (StatSoft, Kraków, Poland). Statistical differences were reported using the Mann-Whitney's $U$ test, with significance set at $p<0.05$.

\section{Results}

A normal distribution of the parameters analyzed between the control and experimental groups (group 1) was confirmed. No difference between the number of women and men was obtained. In group la, the patient age ranged from 35 to 71 years (average 49.1). In group $1 \mathrm{~b}$, the patient age ranged from 18 to 85 years (average 47.8). The difference between the groups was not significant. The difference in bone defect size was also not significant. The Body Mass Index $\left(\mathrm{BMI} ; \mathrm{kg} / \mathrm{cm}^{2}\right)$ values in the groups were comparable. In the skin group, the patient age ranged from 38 to 59 years (average 45.9 ) and all of them were males.

Complications included minimal pain or bleeding associated with donor vein site, which resolved within few hours; a visual examination revealed a smooth submucosal swelling in several patients from experimental group la at the injection site and that was few millimeters in diameter and was resolved over several hours.

In cases where L-PRP was applied, a decrease in bleeding from bone and soft tissue was noted during the operation. In patients with an odontogenic mandibular cyst from experimental group more advanced healing processes of oral mucosa were noted in comparison to cases where the gel was not added. The sutures in L-PRP group were left in the wound for 5 days and the stitches in the control group were removed on the 7th day. In both groups the wound margins were redcolored 7 days after operation, but in the gel group this was less pronounced (Figures 1 and 2). No signs of inflammatory reactions were found at the surgical site after 14 days. 


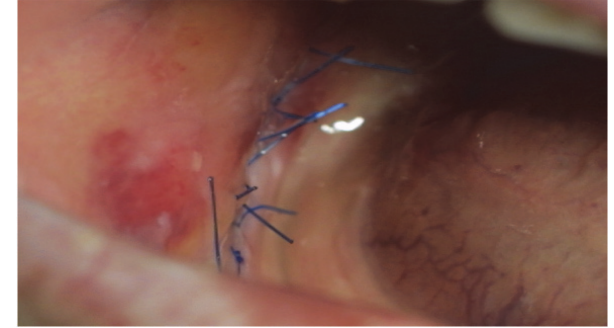

Figure 2: Postoperative wound in the 3rd day after cyst operationexperimental group.

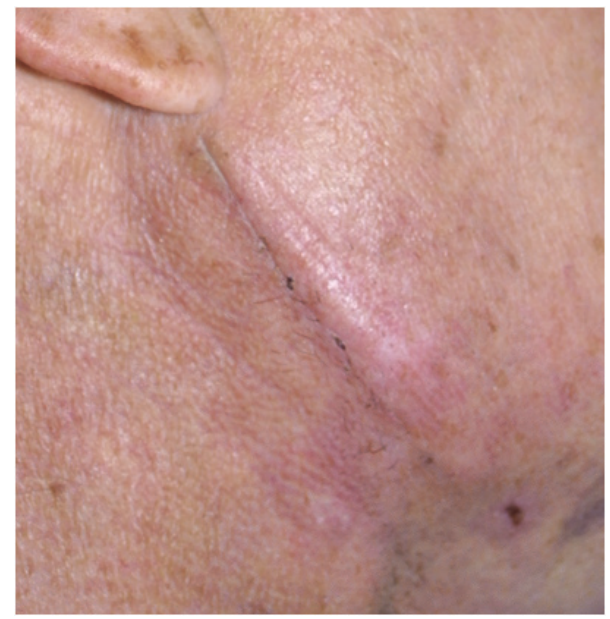

FIgURE 3: Postoperative wound in the 14th day after mandibular fracture fixation-control group.

The average whole blood platelet counts were $223 \pm 69 \times$ $10^{9} / \mathrm{L}$ and $1718 \pm 482 \times 10^{9} / \mathrm{L}$ in L-PRP. The mean leukocyte number was $5,021 \pm 2,001 \times 10^{9} / \mathrm{L}$ in blood and $39,1 \pm 14,1 \times$ $10^{9} / \mathrm{L}$ in L-PRP.

The average hospitalization time was 2.3 days.

No complications related to the surgical technique were noted in the double mandibular fracture group. A small hematoma at the L-PRP site occurred in 2 patients, which resolved over several days. Pain at the L-PRP injection site was relieved within few hours after patients had the operation, which at days 1,2 , and 3 was confirmed by VAS estimation (average 8.2 in control and 4.6 in experimental group). However, there were no differences observed in the healing process progression. The wound margins were redcolored at the same level 7 days postoperatively in both groups (Figures 3 and 4). The stitches were removed on the 7 th day. No bacterial processes complicating wound healing were noted. After 14 days a small line of scar tissue was observed.

The average whole blood platelet counts were $244 \pm 68 \times$ $10^{9} / \mathrm{L}$ and $1830 \pm 489 \times 10^{9} / \mathrm{L}$ in L-PRP. The mean leukocyte number was $5,875 \pm 2,011 \times 10^{9} / \mathrm{L}$ in blood and $45,2 \pm 15,3 \times$ $10^{9} / \mathrm{L}$ in L-PRP.

The average hospitalization time was 3.8 days.

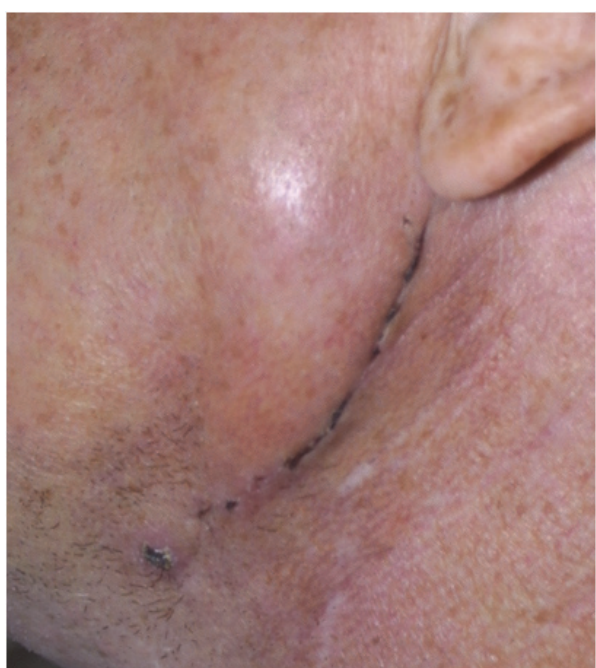

FIGURE 4: Postoperative wound in 14th day after mandibular fracture fixation-L-PRP group.

\section{Discussion}

The use of growth factors in combination with tissue engineering seems to be the most promising future treatment method for skin and soft tissue defects $[3,7]$. The autologous sprayed or percutaneous L-PRP administration that is associated with the classic open grafting technique offers the advantage of decreasing morbidity [3].

The average time for platelet survival in wounds and the onset of releasing growth factors is about 5 days. Elongating the soft tissue healing process is performed in two ways. The first way depends on increasing the number and stimulation of stem cells, which secrete TGF- $\beta$ and IGF to the soft tissue matrix $[4-6,8]$. The second and dominant mechanism acts through chemotaxis and macrophage activation, which after the third day takes over platelet function by secreting growth factors. Macrophages migrate to the site of injury due to PDGF activity and the tissue oxygenation difference becomes higher than $20 \mathrm{mmHg}$, which takes place between the dead space and the adhesive normal oxygenated tissue $[8,9]$. In a typical transplant, hypoxia leads to an oxygenation difference of 30-40 mmHg. When the influence of secreted PDGF by platelets starts to decrease, growth factors which are synthetized by macrophages start to predominate. The growth factors are similar to PDGF, but they are synthesized by macrophages. Stem cells also secrete TGF- $\beta$ and IGF in an autocrine manner, which stimulate healing [8-11].

The importance of platelet derived growth factors in the repair of soft tissue ulcers in clinical studies has been widely documented $[3,7,11]$. Glover et al. carried out a retrospective study evaluating the wound healing and limb salvage outcomes over a 4-year period in 3830 patients [7]. Their study showed that patients treated with comprehensive wound care plus topical use of autologous platelets release had significantly higher rates of wound healing and increased limb salvage than those treated with comprehensive care alone. Recent publications also demonstrated improved outcomes during cardiac surgery when L-PRP was applied 
during closure of the sternal wounds [12]. The incidence of superficial infection in their studies was significantly lower in the inductive gel group (0.3\%) compared to the no L-PRP group $(1.8 \%)$. There was a similar relationship found when comparing deep sternal wound infections (L-PRP, $0.0 \%$, versus no L-PRP, 1.5\%). They concluded that the application of L-PRP in patients undergoing cardiac surgery seems to confer a level of protection against infection, although the mechanisms of action remain to be elucidated. In our experiments, based on clinical examinations, we observed that the oral mucosa in patients from the L-PRP-treated group healed faster than in control patients.

Some authors reported that L-PRP might not produce the desired stimulatory response when autologous bone is not present in the graft or when the defect is of a large volume because vital bone cells are needed for this stimulation to occur [3]. Marx et al. [8] reported that $5 \mathrm{ml}$ of L-PRP with 1 million platelets $/ \mu$ is required to induce bone and soft tissue healing processes. Since we applied L-PRP without bone marrow grafts, we decided to increase the L-PRP volume to $6 \mathrm{ml}$. In our study, the odontogenic cysts were filled up with L-PRP without autologous bone or bone marrow grafts. Nevertheless, the occurrence of bleeding during cyst removal and the wall curettage could induce additional release of growth factors that would stimulate progenitor cells localized in the bone matrix. L-PRP application was sufficient to increase bone and soft tissue healing processes in smaller and bigger cysts despite the lack of osteogenic cells from harvested bone grafts.

In a wound treatment study by Crovetti et al. [13], a reduction in pain sensation was observed when L-PRP was applied, but they offered no explanation for these observations. In Everts et al.'s study [14], the application of L$\mathrm{PRP}$ resulted in reduced postoperative pain, decreased usage of pain medication, and a quicker recovery in shoulder function. Santavirta and coworkers [15] studied shoulder pain originating from the subacromial bursa. They performed an immunohistochemical study in patients operated for OSD (open subacromial decompression) and demonstrated that immunoreactive sensory afferent fibers arise in the subacromial bursa. Interestingly, Sprott et al. [16] reported pain reduction following acupuncture and measured a decrease in serotonin (5-HT) concentration in platelets from these patients and an increase in 5-HT levels in plasma, suggesting a normalization of plasma 5-HT levels due to the mobilization of platelet 5-HT [16]. Everts et al. also noted that, except for the alpha granules, platelets also contain dense granules, which hold large amounts of 5-HT [17]. Since the platelet counts were almost sixfold higher in the L-PRP, 5-HT might have been released at the wound site following platelet activation. Also, a chronic inflammatory reaction has been demonstrated [15], resulting in the activation of nociceptors. These patients developed serious pain complaints [18]. Blaine et al. [19] proposed to use anti-inflammatory agents for the treatment of subacromial impingement, in order to reduce inflammation. In this regard, Everts et al. [14] postulated an additional effect of L-PRP when applied to inflammatory tissues, since we demonstrated the presence of high concentrations of viable leukocytes, particularly neutrophils and monocytes [14]. In addition, platelet growth factors initiate macrophage migration [14]. In general, many leukocytes are playing a key function as regulation turntable of the healing and inflammatory process and they also present antinociceptive effects through different chemokines, antiinflammatory cytokines (IL-4, IL-10, and IL-13), and opioid peptides (b-endorphin, metenkephalin, and dynorphin-A) and can therefore promote a clinically relevant inhibition of pathological pain [20]. In our study in double mandibular fracture patients, based on VAS scores, we noted that pain at the L-PRP site was less intensive and was relieved faster than in the control site.

\section{Conclusions}

As promoters of wound healing, L-PRP and L-PRF are powerful tools for soft tissue reconstruction in cosmetic, plastic, and reconstructive surgery. The use of these materials results in a reduction in operating time and postoperative pain, enhances flap and graft survival, hastens reepithelialization, and decreases the necessity of drains and pressure dressings and the incidences of complications. Like the fibrin glues, LPRP and L-PRF are effective in stopping capillary bleeding in the surgical flaps. Their inflammatory-regulating properties promote edema end ecchymosis reduction. This field of research offers great perspectives for new therapeutic options and requires an extensive evaluation of all the potential clinical methodologies.

\section{Competing Interests}

The authors declare that they have no competing interests.

\section{References}

[1] A. Cieslik-Bielecka, D. M. Dohan Ehrenfest, A. Lubkowska, and T. Bielecki, "Microbicidal properties of Leukocyte- and PlateletRich Plasma/Fibrin (L-PRP/L-PRF): new perspectives," Journal of Biological Regulators \& Homeostatic Agents, vol. 26, pp. 43S52S, 2012.

[2] R. C. Chambers and G. J. Laurent, "Coagulation cascade proteases and tissue fibrosis," Biochemical Society Transactions, vol. 30, no. 2, pp. 194-200, 2002.

[3] A. Cieslik-Bielecka, T. Bielecki, T. S. Gazdzik, T. Cieslik, and T. Szczepanski, "Improved treatment of mandibular odontogenic cysts with platelet-rich gel," Oral Surgery, Oral Medicine, Oral Pathology, Oral Radiology and Endodontology, vol. 105, no. 4, pp. 423-429, 2008.

[4] J.-P. Fréchette, I. Martineau, and G. Gagnon, "Platelet-rich plasmas: growth factor content and roles in wound healing," Journal of Dental Research, vol. 84, no. 5, pp. 434-439, 2005.

[5] H. Li, L. Fredriksson, X. Li, and U. Eriksson, "PDGF-D is a potent transforming and angiogenic growth factor," Oncogene, vol. 22, no. 10, pp. 1501-1510, 2003.

[6] T. Bielecki, A. Cieslik-Bielecka, M. zelawski, and W. Mikusek, "A side-effect induced by the combination of a demineralized freeze-dried bone allograft and leucocyte and platelet-rich plasma during treatment for large bone cysts: a 4-year followup clinical study," Transfusion and Apheresis Science, vol. 47, no. 2, pp. 133-138, 2012. 
[7] J. L. Glover, M. S. Weingarten, D. S. Buchbinder, R. L. Poucher, G. A. Deitrick III, and C. P. Fylling, "A 4-year outcome-based retrospective study of wound healing and limb salvage in patients with chronic wounds," Advances in Wound Care, vol. 10, no. 1, pp. 33-38, 1997.

[8] R. E. Marx, E. R. Carlson, R. M. Eichstaedt, S. R. Schimmele, J. E. Strauss, and K. R. Georgeff, "Platelet-rich plasma: growth factor enhancement for bone grafts," Oral Surgery, Oral Medicine, Oral Pathology, Oral Radiology, and Endodontics, vol. 85, no. 6, pp. 638-646, 1998.

[9] Y. Ogino, Y. Ayukawa, Y. Tsukiyama, and K. Koyano, "The effect of platelet-rich plasma on the cellular response of rat bone marrow cells in vitro," Oral Surgery, Oral Medicine, Oral Pathology, Oral Radiology and Endodontology, vol. 100, no. 3, pp. 302-307, 2005.

[10] G. Sammartino, D. M. Ehrenfest, J. A. Shibli, and P. GalindoMoreno, "Tissue engineering and dental implantology: biomaterials, new technologies, and stem cells," BioMed Research International, vol. 2016, Article ID 5713168, 3 pages, 2016.

[11] T. Bielecki, K. Wójcik, T. Bold, B. Osadnik, and T. Szczepański, "The influence of leukocyte and platelet concentrate enrich in stem cells on bone regeneration processes: a clinical and flow cytometry study," Journal of Biomedical Science and Engineering, vol. 8, no. 9, pp. 659-664, 2015.

[12] C. C. Trowbridge, A. H. Stammers, E. Woods, B. R. Yen, M. Klayman, and C. Gilbert, "Use of platelet gel and its effects on infection in cardiac surgery," Journal of Extra-Corporeal Technology, vol. 37, no. 4, pp. 381-386, 2005.

[13] G. Crovetti, G. Martinelli, M. Issi et al., "Platelet gel for healing cutaneous chronic wounds," Transfusion and Apheresis Science, vol. 30, no. 2, pp. 145-151, 2004.

[14] P. A. Everts, R. J. J. Devilee, C. B. Mahoney et al., "Exogenous application of platelet-leukocyte gel during open subacromial decompression contributes to improved patient outcome. A prospective randomized double-blind study," European Surgical Research, vol. 40, no. 2, pp. 203-210, 2008.

[15] S. Santavirta, Y. T. Konttinen, I. Antti-Poika, and D. Nordström, "Inflammation of the subacromial bursa in chronic shoulder pain," Archives of Orthopaedic and Trauma Surgery, vol. 111, no. 6, pp. 336-340, 1992.

[16] H. Sprott, M. Jeschonneck, G. Grohmann, and G. Hein, "Microcirculatory changes over the tender points in fibromyalgia patients after acupuncture therapy (measured with laserDoppler flowmetry)," Wiener Klinische Wochenschrift, vol. 112, no. 13, pp. 580-586, 2000.

[17] P. A. M. Everts, C. Brown Mahoney, J. J. M. L. Hoffmann et al., "Platelet-rich plasma preparation using three devices: implications for platelet activation and platelet growth factor release," Growth Factors, vol. 24, no. 3, pp. 165-171, 2006.

[18] P. Grigg, H.-G. Schaible, and R. F. Schmidt, "Mechanical sensitivity of group III and IV afferents from posterior articular nerve in normal and inflamed cat knee," Journal of Neurophysiology, vol. 55, no. 4, pp. 635-643, 1986.

[19] T. A. Blaine, Y.-S. Kim, I. Voloshin et al., "The molecular pathophysiology of subacromial bursitis in rotator cuff disease," Journal of Shoulder and Elbow Surgery, vol. 14, no. 1, pp. S84S89, 2005.

[20] D. M. D. Ehrenfest, I. Andia, M. A. Zumstein, C.-Q. Zhang, N. R. Pinto, and T. Bielecki, "Classification of platelet concentrates (Platelet-Rich Plasma-PRP, platelet-rich fibrin-PRF) for topical and infiltrative use in orthopedic and sports medicine: current consensus, clinical implications and perspectives," Muscles, Ligaments and Tendons Journal, vol. 4, no. 1, pp. 3-9, 2014. 

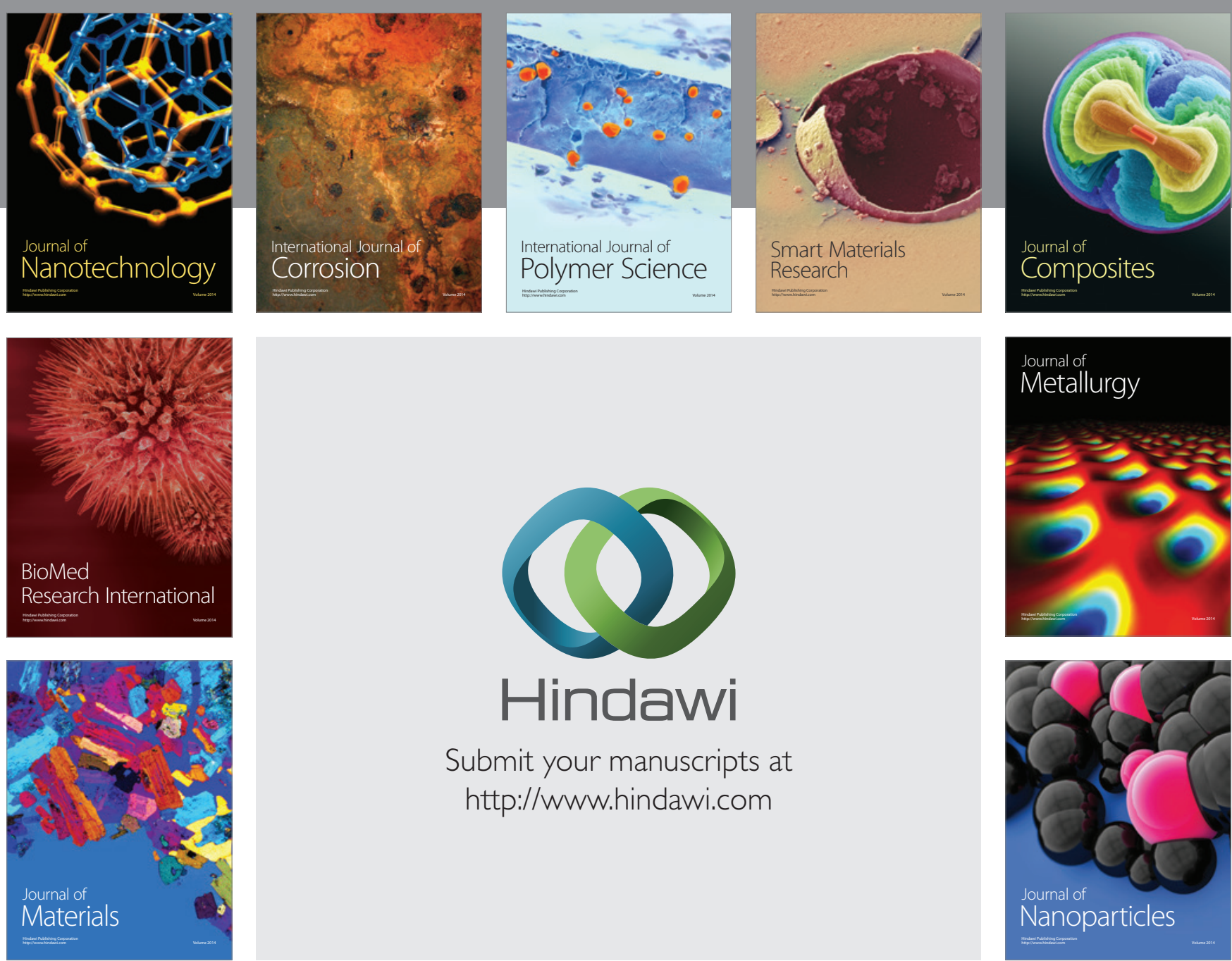

\section{Hindawi}

Submit your manuscripts at

http://www.hindawi.com

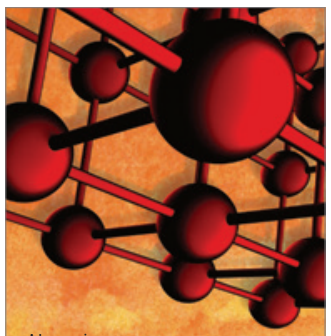

Materials Science and Engineering
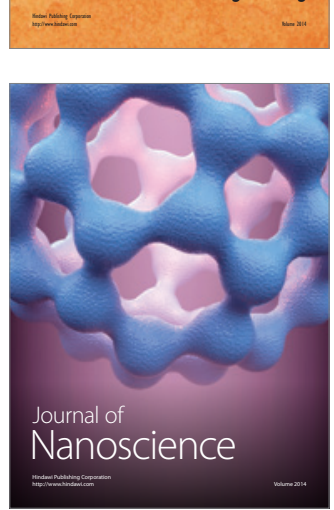
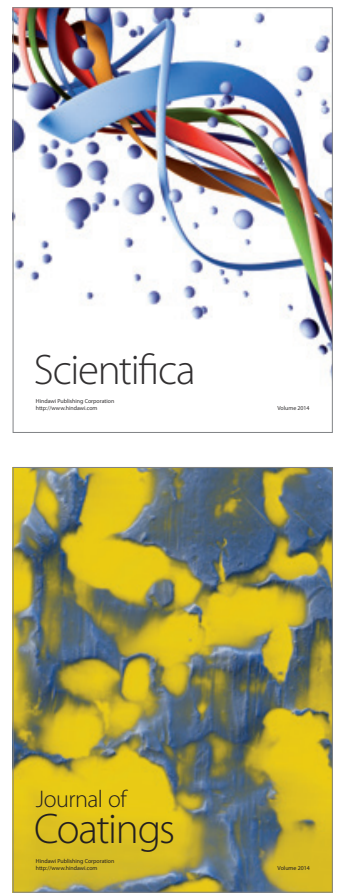
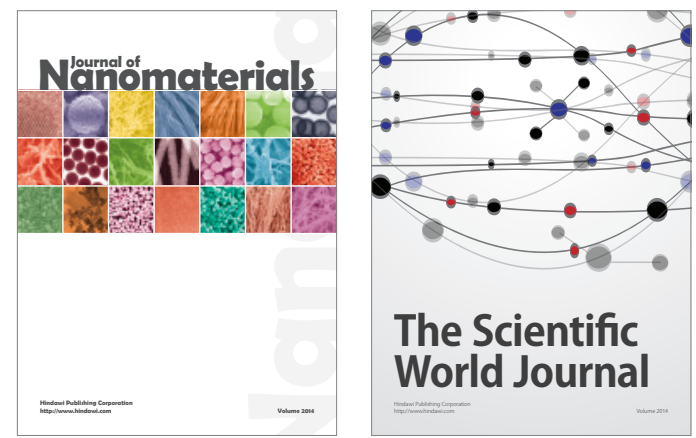

The Scientific World Journal
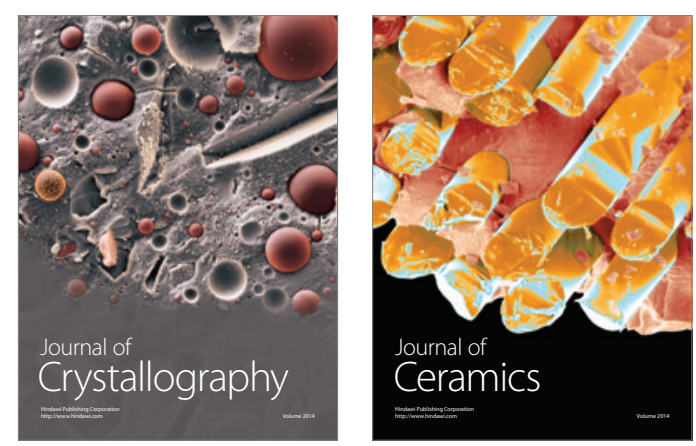
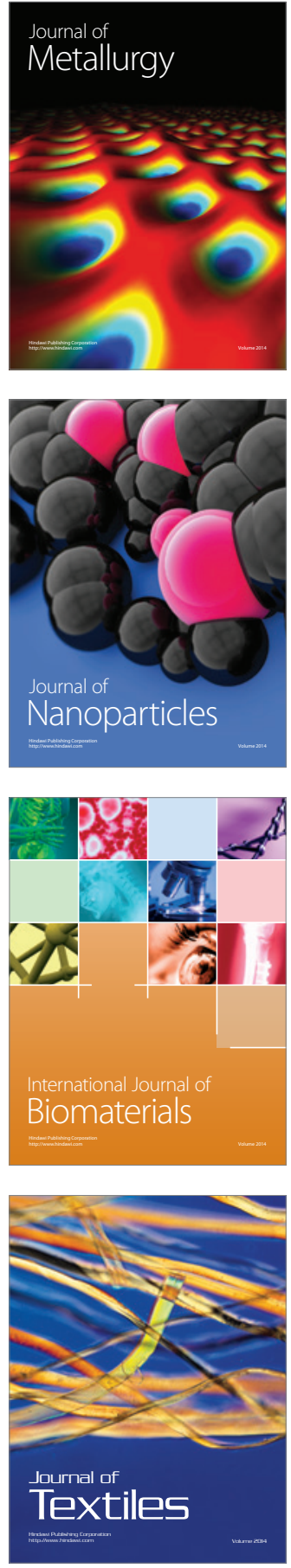